\title{
Symbiotic and Sustainable Design Research for the Indicator System of Icon and Environment In Ancient Village
}

\author{
SHIYAO .DING ${ }^{1} \&$ PING.GU ${ }^{1}$ \\ ${ }^{1}$ Jiangnan University, Wuxi, China
}

\begin{abstract}
KEYWORD: Sustainable Development ;Icon ; Symbol Design ;Symbiotic Design
ABSTRACT: The existence mode of ancient villages is different from urban environment where the commercialization continues to strengthen. The constancy of life produced change with sociological significance from visual expression to evaluation of value since the intervene of new elements.It has been through the reconstruction of historical language from the natural order of villages to compulsory intervention of icon, whereas the reconstruction does not mean put in stiffly. The realistic question is that through set up icon and indicator system having a negative effect on ancient village environment. It is difficult to fusion between modernization of symbols and obsolete architecture. Village unit is a unified symbol from the vision system to daily life. It is the symbiotic design that is contributed to guide the environment and icon design system. Symbiosis represents effective stacking and crossover influences between multi-elements and which reasonably demonstrate on the hierarchy of visual order.

On the basis of understanding and analyzing the using status of icon and indicator system in ancient villages, probing the relationship between ancient village environment and icon design and design problems that spring from it. Ancient villages in paper mainly refer to two ancient villages around the Taihu Lake named Dongcun and Mingyuewan village. The designation of geographical scope is the precondition for research. Natural conditions and architecture style is very important for icon design. The local environment of traditional village is facing constant change of morerniazation, which becoming a sustainable problem for the development of ancient villages and cultural continuity problems.
\end{abstract}

Intervention form that impose icon system to the ancient villages is the core of this thesis. Through the effectiveness that the status quo of a village's icon system and the quantization of icon system have on the guidelines routes, the author sums up three icon design models for the ancient environment. Using sociological and psychological methods to test systematic derivative ideas of icon design, which is beneficial for transforming the research achievements of cases to guide the theoretical development of subjects.

In the icon design which is based on semiology as its theoretical basis, the application of icon design is constantly expanding from public places in cities to non-commercial environment, which put forward more requirements for the principle and theory of icon design. The integral family pattern of ancient villages is a premise of the design. With the diversity of observation styles, the visuallyoriented system of ancient villages is still simple and lack of integrity. With the embedded development of design industry, what icon system can do to help the protection of traditional heritage worthy of attention.

It is difficult to cope effectively with the combination of traditional environment and modern icon system, then, the reason for this is that the difference between design and the main body has not been fully considered. When the design method that suitable for environment introduced to the other environment with different attributes, the purpose and identification of the icon are accordingly changed which refers to changes in the symbolism. The design principles in public environment aim to meet the purpose of business promotion and guide. The theoretical model of semiology plays the role of stimulating when successfully translated during the process of asking the direction. The purpose of the article is dealing with coordination and common design principles of modernization and tradition. 


\section{THE NON-COMMERCIAL PRINCIPLE IN VILLAGE AND COMMERCIALIZATION ATMOSPHERE OF ICON DESIGN}

For the research content, life pattern of farmers who live in the ancient villages have some existing labor law which are divided by the unit. For the protection and tourism development of the ancient village itself, it lacks of focus on the presence of the main village in the icon system. the icon system and design seldom focus on the village itself. There has been alienation phenomenon of the icon in the city where the folk idea of stationing in a place is gradually faded. In other words, the situation has changed from the thing serves people to people to be bound and restricted. So the negative effect of commercialization becomes the problem during the transformation of ancient village. The noncommercial design principle should be taken into account with the retention of original style and features in ancient village. Because of the propagated commercial behavior, space which has not been invaded is especially precious.

Nevertheless, the simple join of the modern element like icon instruction is particularly severe. Under the design guidance of "removing", the icon is not only the decrease in amount but also the transformation of the design methodology. The visual conflict between commercial symbol and the concatenation of ancient villages demonstrates on the disharmony of icon and architecture, due to the nonrelevance in the form.. From the relationship of icon design and the outer environment which consist the whole visual image, "The unique quality of art work equals to the fact that embedded in traditional context. Of course, the tradition itself is vivid and flexible." ${ }^{1}$ From the inspection utility of visual experience, the icons stick in public environment should be conform to the commonly used design value, seeing the icons form the direct and indirect value of visual guidance.

In the process of doing research of Chinese village life, the key element for icon design is basic convention. Chinese village naturally distributed as functional purpose and divided as family unit by aim of production and operation. In ancient villages where restricted by natural conditions, they are based on manual labor and cottage industry work as basic scale before advanced tools and communication conditions have not involved into village. Under this premise, icon designs in natural villages also exist excessive commercialization tendency. During the process the villagers attract the tourists, the icon set up by villagers is also the part of icon. Its commercial properties were more straightforward. On the one hand, it is helpful to the village itself as the route guidance. On the other hand, any icon in the strange environment needs to be explained. Meanwhile, it is the interpretation for the environment and helpful to other people who need to visit the village.

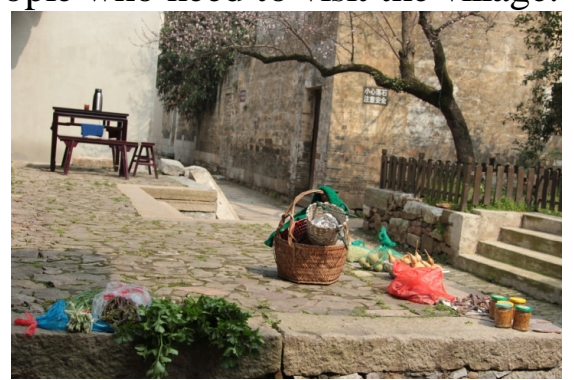

Figure1 Farming items for sailing 


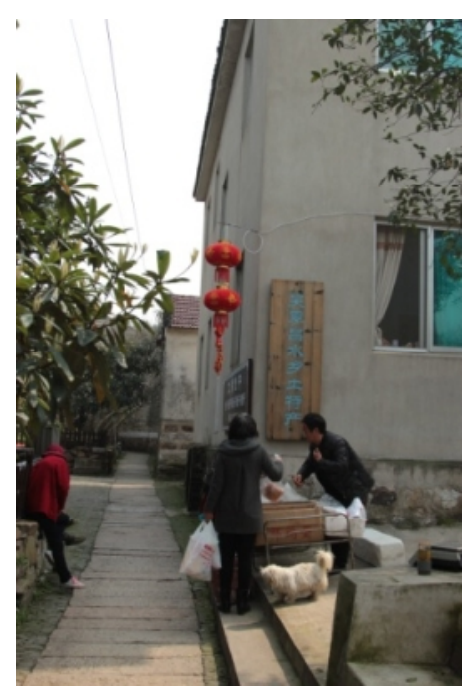

Figure 2 The scene of sailing farming items

Here is a simple division that based on functional aspects, such as warning icon, life service icon and route guidance icon and the introduction icon in commercial scenic spot. Due to the different function of different icons, the location of each icon is different. It is necessary to forbidden to smoke and need to be clarified at the entrance of the old buildings. Undoubtedly some icons are ought to be set out during the construction of the houses where need to indicate the danger. The icon system has the warning effects in villages. And it also plays an important role in protecting buildings and maintaining security. However, there are some non-unified situation in the ancient villages in warning icon system, such as the left side of the blue icon has obvious difference in color and solor with "no smoking" on the right side of the obvious In the same kind of indicator system, there are some deficiency due to the interpretation ambiguity, caused by the chaos of color and form elements. If abandon the interpretation of language, It is certain that there are some obstacles in using other symbol attributes like images to classify and establishing barrier-free design.

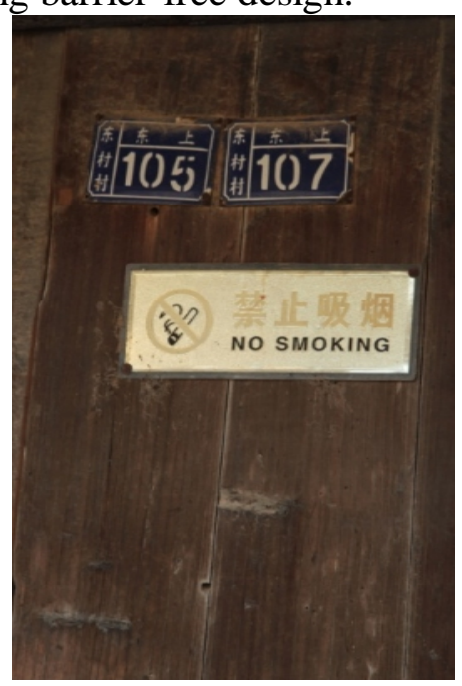

Figure3 Application of icon with warning effect in ancient village

Visual sensory stimulation in city's commercial competition is intensified At the same time, it is surrounded by different levels of icon and resulting in a "blind eye" of visual culture. In the villages that absent of reference, there are coordination problems between the independent icon system and the environment. From graphics, material and output effect of icon system, the system itself that are not consistent with environment is the destruction of design. In cities with commercialization and high degree of civilization, the existing design shall also be used in other environments? Surprisingly, the answer may be yes. The existence of symbol is not independent whereas it is limited by things and cultural ethics, that is acceptance level of people. People's interpretation of the icons is along with the historical development and subtle secondary factors. such as person's identity, gender and purpose behavior and so on. 


\section{THE DEMONSTRATION OF ICON IN SUSTAINABLE DEVELOPMENT IN EXISTING CHAIN}

Icons in ancient village undertake diverse classification function, such as icon of public phone hanging up in store selling items for daily use. In chain of icon formation, it has the basic direction and comprehensive engagement. The function of icons could first be interpreted from the comprehensive relationship of people, events and objects. The event feature of icon expresses the descriptive feature of images by graphic symbols. Compared with symbol design, icon design currently in ancient village conveys information by static and single way. Icon is relatively lack of entertainment. From the means of transmission, it is more traditional, giving priority to vertical plaques. The interpretation way of icon is relatively fixed. The following saying will help differentiate the icons. " Icons are graphic and symbol that free to choose. Symbol is different from icon which allows containing entertainment elements. Meanwhile, icon have some important function by mobile phones, personal handheld computers and other electronic digital media system, especially the device screen resolution is no longer enough to display symbols . " 2

In the life of villagers in ancient villages, the economic value created by daily farming labor is certain. With local natural conditions related industry, it has directly impact on rural industrial scale and improvement of utility. To construct tourism industry of ancient villages by icon, in process of industrial industrialization, the combination between icon design and village environment is worthy to be discussed. There are connection between rural handicraft properties and natural conditions, rural industrialization and the village has a close relationship with artificial environment. For example, there are inner connecting between courtyard environment and village around the courtyard. From the color between environmental space and material texture which are inseparable.

Take kitchen for example, the form and structure of hearth and village of existing energy reserves form necessary relationship. The kitchen which located in the open or half-open yard was placed around the entrance of house. Fuel used in the hearth in old village was mainly used the branches of plants or trees in the field, The method of obtain materials from local sources matched the basic design principle. As far as investigated, the fuel used by the habitants were composed of electricity Igas and branches of plants. The community research means study of culture in a place. Regardless of study is from perspective of history or ethnography, it seems to be the practice field for developing theory and paradigm. Surprisingly, category of space has not been thinking for so long, or as established premise. In spite of this_ or just because of this_- the location in "folklore" on the premise of culture area. A similar phenomenon is in geographical research, as Baosingh pointed out, regional culture research often stay in cultural space order which could not reach behavior and process of cross-examine space culture. In other words, the way of people experience in daily culture space and arrangement of space." 3

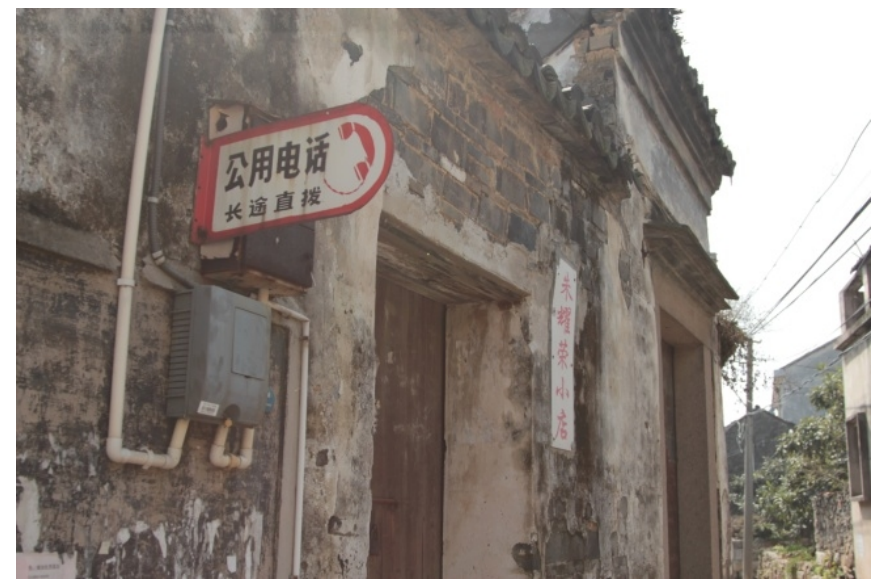

Figure4 Using condition of life service icons 


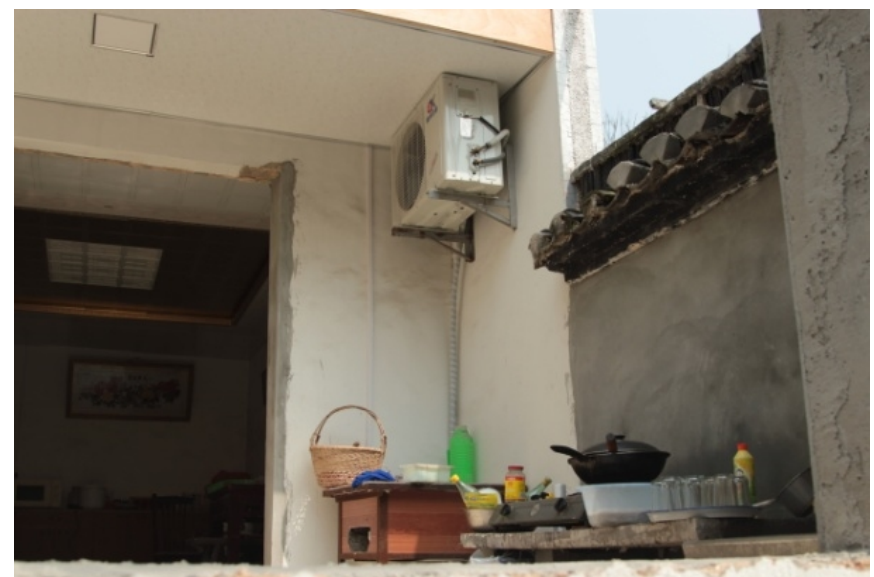

Figure5 Courtyard environment of ancient village

Take Huangshi ancestral hall in Mingyuewan village for instance, it has become a typical case of commercial ancient villages. From the front entrance of architectural form, it basically follows the traditional style. For the convenience of visiting, there are different heights of icon plates set up for introducing and directing icons.

Model is the custom, moreover, also the concept module of icon design. In ancient village where commercial atmosphere is gradually strengthened, the model of the stability of the network life pattern was broken. Model was reconstructed. Icon system is important segment in during reconstruction. Clues which were helpful for recognizing things is the fixed exchange between farmers in villages. Nevertheless, after the intervention of commercialization, the periodic visiting of large amount of strange visitors took replace. The change of survival behavior reflects subtle relationship between design experience and customs. Similar to the ancient village, commercial openness of ancestral temple of Dongcun village in the current times is not yet mature, but the course is basically consistent. Disrepair and man-made destruction viewed as thorny problems for the ancient buildings protection. Also, the intervention of graphic system is also vandalism for the protection of village.

\section{SYMBIOTIC DESIGN OF ICON AND ENVIRONMENT}

Symbiotic has the meaning of mutual participation and environmental construction. "Whether recipients understand the significance of planning, More importantly, It's not just a symbol. By the time this symbol associated with one or the other symbols cases, also could produced different meanings. Context which means interconnected meaning from which the recipients understood by symbols, accordingly, the correct understanding of icon plays a decisive role. " ${ }^{4}$ The context of icon includes usual unit of village, polymerization of family as the basic composition and the natural humanities environment in ancient village and even the cultural and history surrounding of Taihu. While the corresponding context elements mentioned above was matched up, the experience owned by people that come from certain environment are reflected in the icons system.

Consciousness of construction has the function of combing venation effect in the processes of systematism in visual design. Thus, constructed daily life is gradually generated in the deconstruction and reconstruction. Meanwhile, construction and the principle of symbiosis is consistent from semantics to constructing result. Come down to the icon design in ancient villages ought to be that the icon is constructed together with the life reality. After all, construction is not the same as the real world or simply means copy and repetition. The construction idea is suitable for development system. In the cultural version of ancient villages, the construction should keep and develop more of the existing system. Whereas consider by the form, the content of the construction was implicated by the individual phenomenon which has strengthening effect for the daily repeat and usual action. 


\section{CONCLUSION}

Icon design has been widely implanted and used in city commercial environment from the implantation of urban commercial environment while in the countryside and ancient villages with different living and production environment, finding the deviation of existing environment of icons is the premise for how the icon designs get along with each other and achieve the target of sustainable development.

At the same time, the icon system plays a basic recognition utility role in the interaction relationship between the role of icon system on the existing ancient villages and the environment. And the role of icon system in the existing ancient village and the interaction with the environment played a basic recognition utility. Moreover, the humanized design of graphic design provides a precondition for sustainable development.

Symbiosis design emphasizes the close integraty level between the basic elements. Meanwhile, it directly points to a reasonable design order.

\section{REFERENCES}

[1] Chen Yongguo.2009.Visual Culture Studies: A Reader. Bei Jing,BJ: Peking University Press.

[2] Rayan Abdullah, Roger Hubner.2007.Pictogram and Icons. Bei Jing,BJ:China Youth Press.

[3] Hermann Bausinger.2014. The enlightenment of everyday life. Gui Lin,GL:Guangxi Normal University Press.

[4] Rayan Abdullah, Roger Hubner.2007.Pictogram and Icons. Bei Jing,BJ:China Youth Press.

[5] Fund Project: This paper serves as the phased achievement of special funding research project of Product Ideas and Design Culture Research Center in Jiangnan University; Scientific Research Project of Hetao College (general project like philosophy and social science), number: HTXYSY13003. 